\title{
Funikuläre Myelose oder spinale Ischämie?
}

\section{Warum dieser Fall?}

Bei akut und fluktuierend auftretenden spinalen Symptomen wird ursächlich vorrangig eine vaskuläre Erkrankung in Betracht gezogen, die eine aufwendige Diagnostik bis hin zur spinalen Angiografie erfordert. Dennoch sollten auch atypische Manifestationen anderer Erkrankungen wie der funikulären Myelose nicht außer Acht gelassen werden, die bei rechtzeitiger Erkennung rasch und wirksam behandelt werden können.

\section{Einleitung}

\section{$\nabla$}

Neurologische Symptome eines Vitamin$\mathrm{B}_{12}$-Mangels entwickeln sich üblicherweise subakut bis chronisch [1,2]. Akute Verlaufsformen einer funikulären Myelose (FM) wurden in seltenen Fällen nach therapeutischer oder missbräuchlicher Exposition gegenüber $\mathrm{N}_{2} \mathrm{O}$ (Lachgasnarkosen) bei Patienten mit bis dahin latentem Vitamin- $B_{12}$-Mangel beobachtet. Ursächlich wird dafür die irreversible Oxydation des Coenzyms Vitamin $\mathrm{B}_{12}$ durch $\mathrm{N}_{2} \mathrm{O}$ verantwortlich gemacht [3].

Pathologisch-anatomisch finden sich bei der FM v.a. demyelinisierende Läsionen mit spongiöser Degeneration infolge eines Verlustes und einer Schwellung von Myelinscheiden im Bereich der Hinter- und Seitenstränge des zervikothorakalen Rückenmarks, weniger häufig der Hirnnerven, peripheren Nerven und der weißen Substanz des Großhirns. Mit der MRT können myelopathische Veränderungen als symmetrische Signalsteigerung in den zervikalen Hinter- und Seitensträngen dargestellt werden [4].

Treten spinale Symptome akut und in fluktuierender Intensität auf, muss differenzialdiagnostisch vorrangig eine spinale Ischämie erwogen werden, sodass die spinale MRT wie im nachfolgend dargestellten Fall auch eine DWI-Sequenz einschließen sollte.

\section{Falldarstellung \\ $\nabla$}

Bei der bisher gesunden 50-jährigen Patientin waren 2-3 Wochen vor Aufnahme Oberbauchschmerzen aufgetreten. Am Aufnahmetag traten heftige Thoraxschmerzen mit Kollapsneigung hinzu, so dass die Patientin vom Notarzt in die Notaufnahme unter dem Verdacht auf ein akutes Koronarsyndrom gebracht wurde. Während des Transports berichtet die Patientin von wiederholten Erinnerungsverlusten und wiederholten Attacken von Bewegungsunfähigkeit und Taubheitsgefühl beider Beine.

Bei der Aufnahmeuntersuchung waren die Beine schlaff plegisch bei seitengleich normaler Sensibilität und erhaltenen BER. Innerhalb weniger Minuten besserte sich die Kraft der Beine, sodass diese wieder abgehoben werden konnten und das Stehen und Gehen mit Unterstützung durch 2 Hilfskräfte möglich war. Blasenentleerungsstörungen lagen bei normalem Restharn nicht vor.

Zu diesem Zeitpunkt waren Herzfrequenz und Blutdruck sowie der internistische Befund normal.

Im Labor fielen eine makrozytäre Anämie (Hb 10,9, MCV 115,0fl) bei erniedrigtem Vitamin- $B_{12}$-Spiegel von $<30 \mathrm{pg} / \mathrm{ml}$ und normalem Folsäurespiegel von 12,9 ng/ml auf. Die Antikörper gegen Parietalzellen im Serum waren hochpositiv mit Nachweis einer Typ-A-Gastritis in der Magenschleimhautbiopsie. Die Serum-TroponinWerte und die Liquordiagnostik waren normal.

Die spinale MRT-Untersuchung der HWS und oberen BWS (1,5T-MRT, Avanto, Siemens, Erlangen/Deutschland) zeigte in den T2-gewichteten Sequenzen symmetrisch in den Hintersträngen auf Höhe des Bandscheibenfachs BWK 6/7 eine kurzstreckige Signalsteigerung ohne expansiven Charakter ( Abb.1a und - Abb.1b). In den kontrastmittelgestützten Sequenzen wies die Läsion keine Kontrastmittelaufnahme auf. In der zusätzlich durchgeführten DWI (EPI, b-Wert 1000; - Abb.1c) zeigte sich korrespondierend in der b1000 eine deutliche spinale Signalanhebung ( $\bullet$ Abb.1c) ohne Signalabsenkung in der ADC-Map ( $\bullet$ Abb. 1d). Unauffällig blieben ein cMRT und die zum Ausschluss einer Aortendissektion durch- geführte CTA der Aorta und der hirnversorgenden Arterien wie auch ein EKG, Langzeit-EKG und die transösophageale Echokardiografie.

Das initial durchgeführte EEG zeigte als Grundrhythmus einen Alpha-Beta-Mischtyp ohne herdförmige Veränderungen oder epilepsietypische Potenziale.

Die SEP nach Stimulation des N. tibialis zeigten verzögerte L1-Antworten bei normaler zentraler Leitungszeit. Die MEP ergaben bei Ableitung vom M. abductor hallucis brevis normale Latenzen und Amplituden.

In den ersten 2 Tagen des stationären Aufenthalts traten mehrfach ähnliche Episoden auf, die aber nach Vitamin- $B_{12}$-Substitution (1000 Aqua-Cytobion/d i.m.) sistierten, sodass die Patientin 2 Wochen nach Aufnahme wieder ohne neurologische Defizite entlassen werden konnte und bei einer Verlaufskontrolle 4 Monate später beschwerdefrei war bis auf ein anhaltendes Übelkeitsgefühl trotz Pantoprazoleinnahme. Der neurologische Befund war unauffällig.

\section{Diskussion}

Welche Faktoren im vorliegenden Fall zum Auftreten dieses atypisch akuten Verlaufs der FM durch eine Vitamin- $\mathrm{B}_{12^{-}}$ Resorptionsstörung geführt haben, bleibt ungeklärt. Ähnlich stark erniedrigte Serumspiegel von Vitamin $\mathrm{B}_{12}$ wurden in anderen Untersuchungen auch bei subakutchronischen Verläufen einer FM beobachtet (1). Die Frage, ob die durch den $\mathrm{B}_{12^{-}}$ Mangel veränderten Konzentrationen wichtiger Metaboliten wie Methylmalonsäure, Homozystein und der biologisch aktiven Metaboliten von Vitamin $B_{12}$, Methyl-vitamin B12 und 5'-deoxy-5'-adenosylcobalamin oder begleitende andere Faktoren wie Mangel an Kupfer eine Rolle für den zeitlichen Verlauf und die Schwere der Erkrankung spielen, kann aktuell nicht beantwortet werden [5]. Eine zusätzliche Noxe durch Exposition gegenüber $\mathrm{N}_{2} \mathrm{O}$ war im aktuellen Fall auszuschließen. Als seltene und atypische Manifestation eines Vitamin- $\mathrm{B}_{12}$-Mangels wird in der Literatur auf venöse Thrombosen in atypischer Lokalisation wie etwa der zerebralen Venen [1] hingewiesen 


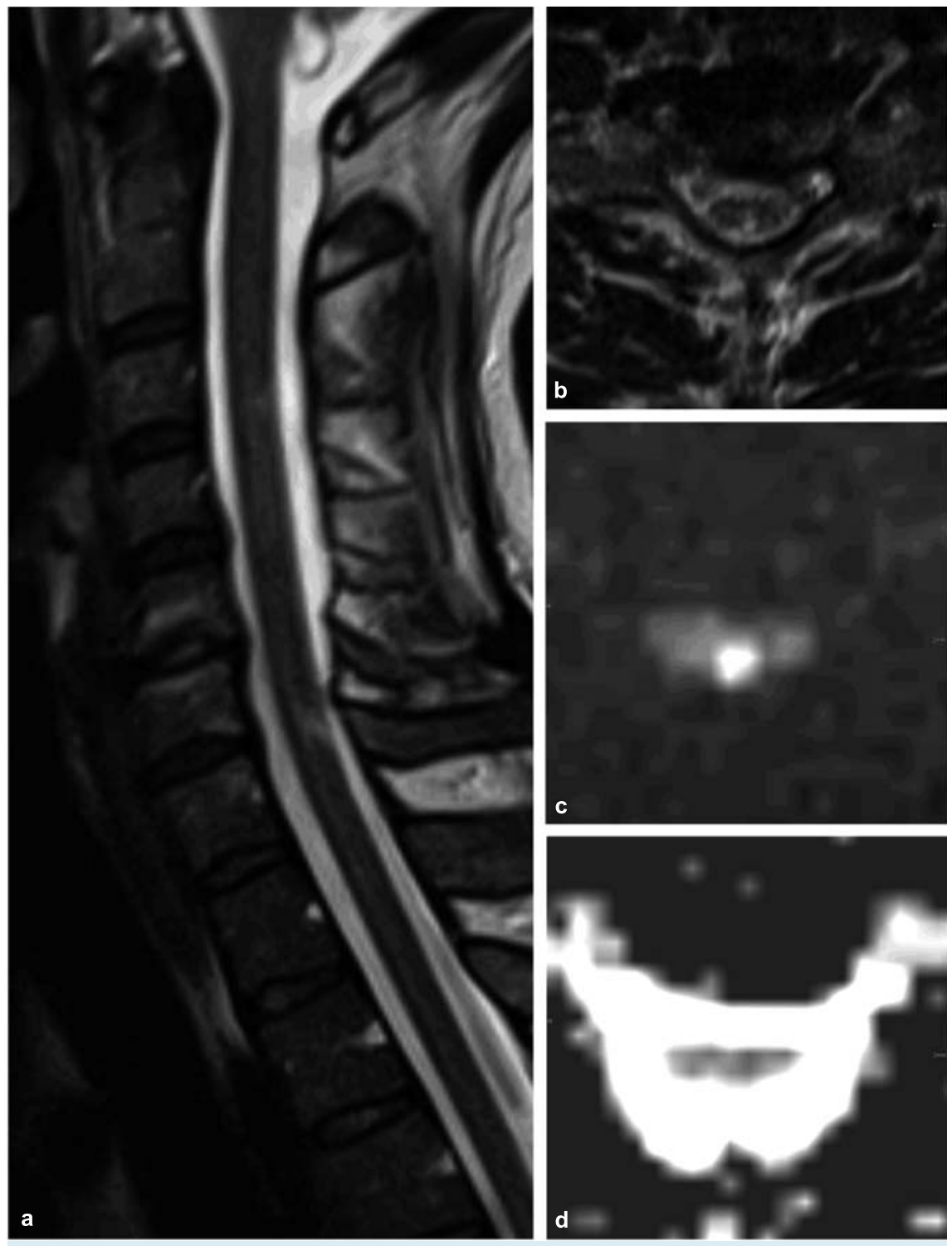

Abb.1 Sagittale (a) und transversale (b) T2 TSE zeigen auf Höhe des Bandscheibenfachs HWK 6/7 eine kurzstreckige hyperintense Läsion beidseits im Hinterstrang ohne raumfordernden Charakter. Die Läsion kommt in der B1000 der DWI (c) stark signalangehoben zur Darstellung, weist in der ADC-Map (d) jedoch weder eine eindeutige Signalabsenkung noch ein T2 shine through auf.

und ein ursächlicher Zusammenhang mit exzessiv hohen Serumhomozysteinspiegeln hergestellt [1].

In zahlreichen Fallberichten zur funikulären Myelose (FM) ist eine häufig längerstreckige intramedulläre Signalsteigerung in den Hintersträngen, seltener den Seitensträngen des zervikalen oder thorakalen Myelons, als Korrelat der o.g. pathologisch-anatomischen Anomalien beschrieben. Als Ursache der Signalanhebung in der T2w ist somit im Akutstadium die intra- und extrazelluläre Ödembildung anzunehmen [1].

Über das Erscheinungsbild der FM in der DWI existieren nach Kenntnis der Autoren lediglich 2 Fallberichte mit chronischem Verlauf einer FM, in denen eine Dif- fusionsstörung in den spinalen Läsionen bei funikulärer Myelose beschrieben ist $[6,7]$. Im Gegensatz hierzu findet sich bei der vorgestellten Patientin bei deutlicher Signalsteigerung in der B0 und B1000 keine Signalabsenkung in der ADC-Map. Daher liegt a.e. analog der Befunde am Gehirn bei einer Kombination aus Diffusionsrestriktion durch intrazelluläre Ödembildung und Diffusionssteigerung durch extrazelluläre Ödembildung eine sogenannte Pseudonormalisierung in der ADC-Map [8] vor.

Differentialdiagnostisch wurde aufgrund des akuten Beginns eine spinale Ischämie diskutiert. Das Auftreten einer isolierten Hinterstrangischämie ist jedoch sehr selten [9], zudem war aufgrund des klini- schen Verlaufs mit rascher Rückbildung der Hypästhesien unter Vitamin- $\mathrm{B}_{12}$-Substitution eine ischämische Genese im Verlauf sehr unwahrscheinlich. Weiterhin wurde wegen der Möglichkeit venöser Thrombosen in atypischen Lokalisationen eine venöse Infarzierung in Betracht gezogen. Venöse Infarzierungen im Myelon sind im Rahmen einer venösen Kongestion bei spinaler duraler arteriovenöser Malformation (nekrotisierende Myelitis, Fox-Alajouanine-Syndrom) beschrieben. Eine solche venöse Kongestion ist aber aufgrund des fokalen Befallsmusters nicht anzunehmen. Identische klinische und radiologische Befunde sind bei Kupfermangel beschrieben [10]. Wegen der Laborkonstellation und der raschen Besserung unter Vitamin- $\mathrm{B}_{12}$-Substitution konnte ein Kupfermangel als Ursache der Beschwerden ausgeschlossen werden. Aufgrund der Liquordiagnostik und des klinischen Verlaufs war auch eine spinale Manifestation einer multiplen Sklerose, bei der MR morphologisch und in der DWI ein identisches Signalverhalten beschrieben ist [11], sehr unwahrscheinlich.

Zusammenfassung Die funikuläre Myelose kann sich atypisch mit akuter und rasch fluktuierender spinaler Symptomatik manifestieren, sodass diagnostisch die Abgrenzung von einer spinalen Ischämie erforderlich ist. Die DWI kann bei akut einsetzender Symptomatik möglicherweise in Ergänzung zur konventionellen MR-Bildgebung die Differenzierung zwischen spinaler Infarzierung und funikulärer Myelose ermöglichen. Allerdings ist die DWI noch kein etabliertes Verfahren zur Erfassung spinaler ischämischer Läsionen, sodass noch weitere Erfahrungen gesammelt werden müssen.

\section{Was lernt man daraus?}

Die funikuläre Myelose kann sich atypisch mit akuter und rasch fluktuierender spinaler Symptomatik manifestieren. Die spinale DWI-Diagnostik kann bei der Differenzierung zwischen spinaler Ischämie und FM helfen, ist aber noch kein etabliertes Verfahren für die Diagnostik spinaler Ischämien.

Interessenkonflikt: Die Autoren geben an, dass kein Interessenkonflikt besteht. 


\section{Literatur}

1 Stabler SP. Clinical practice. Vitamin B12 deficiency. N Engl J Med 2013; 368: 149 160

2 Hengst $S$, Cordes $P$, Götzinger $R$ et al. Funikuläre Myelose: Verlaufsdokumentation von Klinik und spinalen MRT-Veränderungen nach Vitamin-B12-Substitution bei $6 \mathrm{~Pa}$ tienten. Akt Neurol 2004; 31: 333 - 337

3 Takacs J. [N2O-induced acute funicular myelosis in latent vitamin B 12 deficiency]. Anasthesiologie, Intensivmedizin, Notfallmedizin, Schmerztherapie: AINS 1996; 31: 525-528

4 Sen A, Chandrasekhar K. Spinal MR imaging in Vitamin B12 deficiency: Case series; differential diagnosis of symmetrical posterior spinal cord lesions. Annals of Indian Academy of Neurology 2013; 16: 255-258

5 Gursoy AE, Kolukisa M, Babacan-Yildiz G et al. Subacute Combined Degeneration of the Spinal Cord due to Different Etiologies and Improvement of MRI Findings. Case reports in neurological medicine 2013; 2013: 159649

6 Tian C. Hyperintense signal on spinal cord diffusion-weighted imaging in a patient

\section{Möchten Sie von einem besonderen Fall aus Ihrer Klinik berichten?}

Autorenhinweise finden Sie unter: http://www.thieme.de/de/klinische-neurophysiologie/ autoren-1869.htm

Schicken Sie Ihre Beiträge an: k.wessel@klinikum-braunschweig.de. with subacute combined degeneration. Neurology India 2011; 59: 429-431

7 Kim EY, Lee SY, Cha SH et al. Subacute combined degeneration revealed by diffusionweighted imaging: a case study. Clinical neuroradiology 2013; 23: 157-159

8 Lansberg MG, Thijs VN, O'Brien MW et al. Evolution of apparent diffusion coefficient diffusion-weighted, and T2-weighted signal intensity of acute stroke. AJNR American journal of neuroradiology 2001; 22: 637 644

9 Vuillier F, Tatu L, Camara A et al. Unusual sensory disturbances revealing posterior spinal artery infarct. Case reports in neurology 2012; 4: 23-27

10 Kumar N, Ahlskog JE, Klein CJ et al. Imaging features of copper deficiency myelopathy: a study of 25 cases. Neuroradiology 2006; 48: $78-83$

11 Andre JB, Zaharchuk G, Saritas E et al. Clinical evaluation of reduced field-of-view diffusion-weighted imaging of the cervical and thoracic spine and spinal cord. AJNR American journal of neuroradiology 2012; 33 $1860-1866$

\section{Autoren \\ K. Pfadenhauer ${ }^{1}$, F. Runck ${ }^{2}$, A. Berlis ${ }^{2}$ \\ Institute \\ ${ }^{1}$ Neurologische Klinik, Klinikum Augsburg \\ ${ }^{2}$ Abteilung für Diagnostische und Interventionelle Radiologie und Neuroradiologie, Klinikum Augs- burg}

Korrespondenzadresse

Dr. Karl Pfadenhauer

Neurologische Klinik

Klinikum Augsburg

Stenglinstraße 2

86156 Augsburg

karl.pfadenhauer@klinikum-augsburg.de

\section{Bibliografie}

Dol http://dx.doi.org/

10.1055/s-0034-1387636

Online-Publikation: 13.4.2015

Klin Neurophysiol 2015; 46: 100-102

(c) Georg Thieme Verlag KG

Stuttgart · New York

ISSN 1434-0275 